Preprint. Online early access available at: https://esiculture.com/an-infectious-curiosity-morbidcuriosity-and-media-preferences-during-a-pandemic

\title{
An Infectious Curiosity: Morbid Curiosity and Media Preferences During a Pandemic
}

\author{
Coltan Scrivner ${ }^{1,2}$ \\ ${ }^{1}$ Department of Comparative Human Development, The University of Chicago; Chicago, Illinois \\ ${ }^{2}$ The Institute for Mind and Biology, The University of Chicago; Chicago, Illinois
}

\begin{abstract}
In this study conducted during the 2020 Coronavirus pandemic, I explored how trait morbid curiosity was related to interest in 1) factual information about Coronavirus that was specifically morbid, 2) general factual information about Coronavirus, 3) pandemic and virus genres of films and TV shows, and 4) genres of film and TV shows that center around threat more broadly. Participants $(n=125)$ who scored high in morbid curiosity reported increased interest, compared to usual, in pandemic/virus genres as well as horror and thriller genres. Morbidly curious participants were also more interested specifically in morbid information about Coronavirus. Furthermore, disgust sensitivity was unrelated to these preferences. These results provide initial evidence that trait morbid curiosity can predict particular media preferences in the face of a real threat, and that morbid curiosity may reflect an adaptive predisposition in some individuals toward learning about the dangerous and disgusting aspects of a threat.
\end{abstract}

Keywords: Pandemic, Morbid Curiosity, Disgust, Threat, Horror 


\section{INTRODUCTION}

Just three months into the 2020 Coronavirus (COVID-19) outbreak, Contagion, a 2011 movie about a deadly viral pandemic, rose from the $270^{\text {th }}$ most-watched Warner Bros. to the second most-watched Warner Bros. film (Mack 2020). Google Trends shows that the biggest spike in Google searches for Contagion began on March $11^{\text {th }}$ - the same day that US President Donald Trump announced a travel ban on Europe due to the Coronavirus spread - and peaked on March $14^{\text {th }}$ - the day President Trump extended the travel ban to the UK. The 1995 viral pandemic movie Outbreak experienced a similar trend, with Google searches for the film peaking on March $15^{\text {th }}$. Google searches for topics such as "epidemic," "pandemic," "virus," and "Coronavirus" all also peaked that same week (Google Trends 2020). We might reason that these search terms spiked in popularity because people were trying to learn more about the Coronavirus outbreak in response to its recent impact on their daily life around that time. The shutting of international borders may have signaled to the American consciousness that Coronavirus was, in fact, a real threat.

Still, it is puzzling that people would search for entertainment about the topic that was causing mass disruption in their lives. This behavior appears even stranger in light of the fact that pathogenic cues often activate the behavioral immune system, which produces feelings of disgust and motivates avoidance behaviors in response to potentially pathogenic material (Schaller 2011). When we see, smell, or inadvertently touch potentially pathogenic material, we recoil and avoid it; if we take a bite out of spoiled food, we spit it out. This response to potentially pathogenic material is powered by the emotion of disgust, which motivates an organism to behave in ways that minimize infection risk, usually through avoidance. However, a closer look at how the human mind deals with potentially dangerous information may help answer the question of why some people seek out pathogen-related entertainment in the wake of a real pathogenic threat. Indeed, this morbidly curious behavior might make sense as an output of evolved mechanisms that process threatening or dangerous material in organisms cognitively equipped with the ability to imagine themselves in situations and learn from those imagined experiences.

Perceiving potentially pathogenic material should not activate disgust alone. If it did, it would sometimes lead to maladaptive behavior. For example, sexual reproduction involves interaction with bodily fluids, which are typical elicitors of disgust. Disgust at the sight of bodily fluids would lead to obvious reproductive issues if it led to avoidance every time. However, sexual arousal overrides the disgust signals that we are exposed to during sex by specifically lowering sexual disgust and increasing motivation for sex (Ariely and Loewenstein 2006; Stevenson, Case, and Oaten 2011). In other words, decision-making about engagement with potentially pathogenic material must take into account the trade-off between potential dangers and potential benefits (Tybur, Kurzban, Lieberman, and DeScioli 2013). While sexual disgust has been investigated, other systems that may counter or down-regulate disgust are underexplored. Curiosity is one possible counterweight to the typical avoidance behaviors that result from cognitive mechanisms that process pathogen cues.

Though there is little psychological research on the topic, morbid curiosity is a widely recognized phenomenon that is typically described as an interest or curiosity about unpleasant things related to death. While it has been documented that humans in general have attentional biases towards threat (Öhman and Mineka 2001; Scrivner et al. 2019), some people might be especially interested in learning about dangerous phenomena beyond a mere attentional bias. For example, some individuals are more interested in seeing, reading, or otherwise learning about 
topics related to death, violence, and disgust (Zuckerman and Litle 1986; Hoffner and Levine 2005; Ibarra and Maestripieri 2017; Oosterwijk 2017; Scrivner 2020). At first glance, the existence of morbidly curious behavior like this appears to contradict the hypothesis that humans possess a behavioral immune system that motivates them to avoid disgusting material. However, in some people, disgusting and frightening features of the environment do not inspire avoidance, but instead stimulate curiosity. How do we reconcile the findings that people avoid disgusting and frightening material in some cases with the fact that they seek it out as entertainment in other cases?

I argue that individual differences in trait morbid curiosity lead to differences in media and entertainment preferences. Morbid curiosity might be conceptualized as an interest in information that typically inspires avoidance by virtue of being a possible threat to human life. Topics of morbid curiosity, then, are factors that are perceived to lead to or be informative of death. Examples may include the motivations of dangerous people (e.g., true crime), violations of the body (e.g., infections and injuries), violent acts (e.g., videos of murder), and even apparent danger that stems from supernatural sources (e.g., curses, spirits; Scrivner 2020). Instead of completely avoiding apparently dangerous or disgusting features of the world, morbid curiosity rouses internal motivation to explore these features. When a dangerous or disgusting phenomenon is perceived to be near or impending, curiosity may surge in some individuals in order to prompt them to gather information about the phenomenon. Taking the risk of gathering information by briefly exposing oneself to a dangerous phenomenon could be advantageous if it helps the individual know how to deal with the threat in the future or provides unique knowledge. If an individual's local ecology is giving cues that dangerous and disgusting features are present, it could be adaptive to gather some information about these features, even if it requires some exposure to them. If you live in a violent neighborhood, it is good to know how, why, when, and from whom violence erupts. If you live in a pathogen-dense ecology, it is important to know something about the pathogens, such as what infected material looks and smells like. Having some curiosity about these features could lead to an adaptive edge by creating knowledge about how to predict and properly deal with them in future encounters.

Of course, costs and benefits of interacting with morbid material are still calculated, even in morbidly curious individuals. For example, how important is the information? Can you seek it out without endangering yourself? Will you have the chance to learn about it again? In many real-world instances, the costs of obtaining information about dangerous phenomena are high. One way to reduce the risk of interacting with a dangerous phenomenon is to create physical distance between yourself and the dangerous phenomenon. Because the human mind is equipped with the ability to create "simulated" experiences through imagination, humans are able to obtain information about different scenarios with very little risk. The learning potential of such imagined scenarios is especially powerful when it is combined with the ability to transmit these scenarios through oral, written, and re-enacted stories that allow others to learn from them. By listening to, reading, or watching simulations of experiences in the form of fiction media, humans can empathize with and imagine themselves in the positions of characters in stories. By doing so, humans can create meaning and knowledge about similar scenarios in their own lives. Fiction media can also provide an opportunity to try out different strategies to solve problems, including making bad decisions without having to pay a price for them. Indeed, the very function of fiction could be to create compelling simulations of experiences from which important information can be gleaned and transmitted (Mar and Oatley 2008). Just as we may play with materials or ideas in order to make sense of them, we might "play" with simulated scenarios in 
order to learn about the situations they present (Piaget 1945; Clasen, Kjeldgaard-Christiansen, and Johnson 2018; Morin, Acerbi, and Sobchuk 2019). Because humans can learn about dangerous phenomena through simulations, the costs of learning about dangerous phenomena can be low. Assuming the information is accurate and applicable, the benefits of learning about the dangerous information would remain high. Thus, human decision-making likely evolved to process simulations of dangerous and disgusting phenomena as less threatening and/or to feel greater curiosity about this material (i.e., morbid curiosity).

Still, there exist individual differences in the propensity to experience simulations of dangerous and disgusting phenomena without much distress. One example of this is recreational horror. Seeing a masked killer wielding a butcher knife in real life would be terrifying and truly dangerous - nobody would wish themselves in this scenario. But, put this scenario on a screen, and some people will pay to vicariously experience this simulated scenario. If something can be gained from the simulated experience, such as knowledge about how one might act if such a situation were to occur in real life, then the benefits remain large. However, by recognizing the scenario as fiction, the costs are processed as being lower than if it was occurring in real life. This provides a unique opportunity to gather valuable information that would otherwise be dangerous to obtain (e.g., what is it like to be chased by a man with a butcher knife?). At the level of the individual media user's psychology, the curiosity felt about this situation will often overcome the low cost of feeling a bit afraid, resulting in a motivation to seek out this information, even at a (monetary) cost. Thus, variations in levels of trait morbid curiosity may motivate some individuals to become interested in a potentially dangerous or disgusting feature by providing them with an extra spark of motivation that overcomes the perceived cost of interacting with the feature or a simulation of it. Under this framework of simulated experience providing an opportunity for learning and meaning-making, the sudden popularity of pandemic films in the face of an actual pandemic begins to make sense. Specifically, people may become more interested than usual in news and fiction about pandemics due to the presence of a real pathogenic threat, and this interest may be amplified among more morbidly curious individuals.

In this study, I investigated how individual differences in trait morbid curiosity relate to media preferences in the midst of the 2020 Coronavirus pandemic. In particular, I explored whether trait morbid curiosity was related to an increased interest in 1) factual information about Coronavirus that was specifically morbid, 2) more general factual information about Coronavirus, 3) pandemic/virus genres of films and TV shows, and 4) genres of film and TV shows that feature threat more broadly, such as horror and thrillers. This design helps to clarify whether or not trait morbid curiosity amplifies information gathering in response to a threatening aspect of the environment, whether this amplification is specific to factual news about that aspect or also extends to fiction about that aspect, and whether the increase in morbid curiosity is specific to the threatening aspect that is salient or if an increase in morbid curiosity extends to threat-related information more generally. Because Coronavirus is a pathogenic threat, I also explored whether or not trait disgust sensitivity influenced these media preferences and how this might relate to morbid curiosity.

\section{METHOD}

Participants ( $\mathrm{n}=126,62$ female) were recruited through Prolific for a study on personality and news consumption. Prolific is an online recruitment tool that provides a diverse sample pool, high quality participant data, and fair pay compared to other platforms (Peer, Brandimarte, Samat, and Avquisti 2017). The data were collected on March 22, 2020. 


\section{Measures}

Participants first completed the Morbid Curiosity Scale (Scrivner 2020) and the Disgust ScaleRevised (Haidt, McCauley, and Rozin 1994; revised by Olatunji et al. 2007). They then answered 10 questions about their interest in Coronavirus, one question about how threatened they felt by Coronavirus, and reported their current interest (compared to usual) in six different genres of film and TV shows: scary/supernatural, mystery/thriller, pandemic/virus, romance, adventure/action, and comedy (Table 1).

\section{Analyses}

Using the psych package in R (Revelle 2018), exploratory factor analysis was conducted to explore underlying factors in the 10 questions that assessed interest in different aspects of Coronavirus. Cronbach's alpha was used to assess the internal consistency of the factors. Dependent variables used in analysis were the subscales produced from the factor analysis, the question about how threatened the participant felt by Coronavirus, and the genre interest questions. Linear regression models were conducted for each dependent variable controlling for participant sex and age. For each dependent variable, two models were conducted: one with disgust sensitivity as a predictor and one with morbid curiosity as a predictor. For the Coronavirus interest subscales, an additional model was conducted that included how threatened participants felt by Coronavirus. One participant was removed from the regression analyses for reporting something other than male or female for sex.

\section{RESULTS}

\section{Descriptive Statistics}

Participant age ranged between 18 and 72 years $(M=32, S D=11.5)$, with most falling between 18 and 50. Means and standard deviations for responses to each media and Coronavirus question can be found in Table 1.

\section{Morbid curiosity and genre interest}

As expected, trait morbid curiosity was positively correlated with more interest in watching scary/supernatural $(\beta=0.71, S E=0.13, p<.001)$, mystery/thriller $(\beta=0.37, S E=0.13, p=$ $.003)$, and pandemic/virus $(\beta=0.38, S E=0.17, p=.030)$ movies and TV shows in the next week compared to usual (Figure 1). Morbid curiosity was unrelated to interest in watching romance $(p$ $=.778)$, comedy $(p=.291)$, or adventure/action $(p=.091)$ films and TV shows compared to usual (Table 3).

\section{Disgust sensitivity and genre interest}

Disgust sensitivity was unrelated to interest in watching any genre more than usual (all $p$ 's $>.19)$ except for adventure/action, which garnered less interest by those high in disgust sensitivity $(\beta=$ $-0.51, S E=0.20, p=.011)$. 


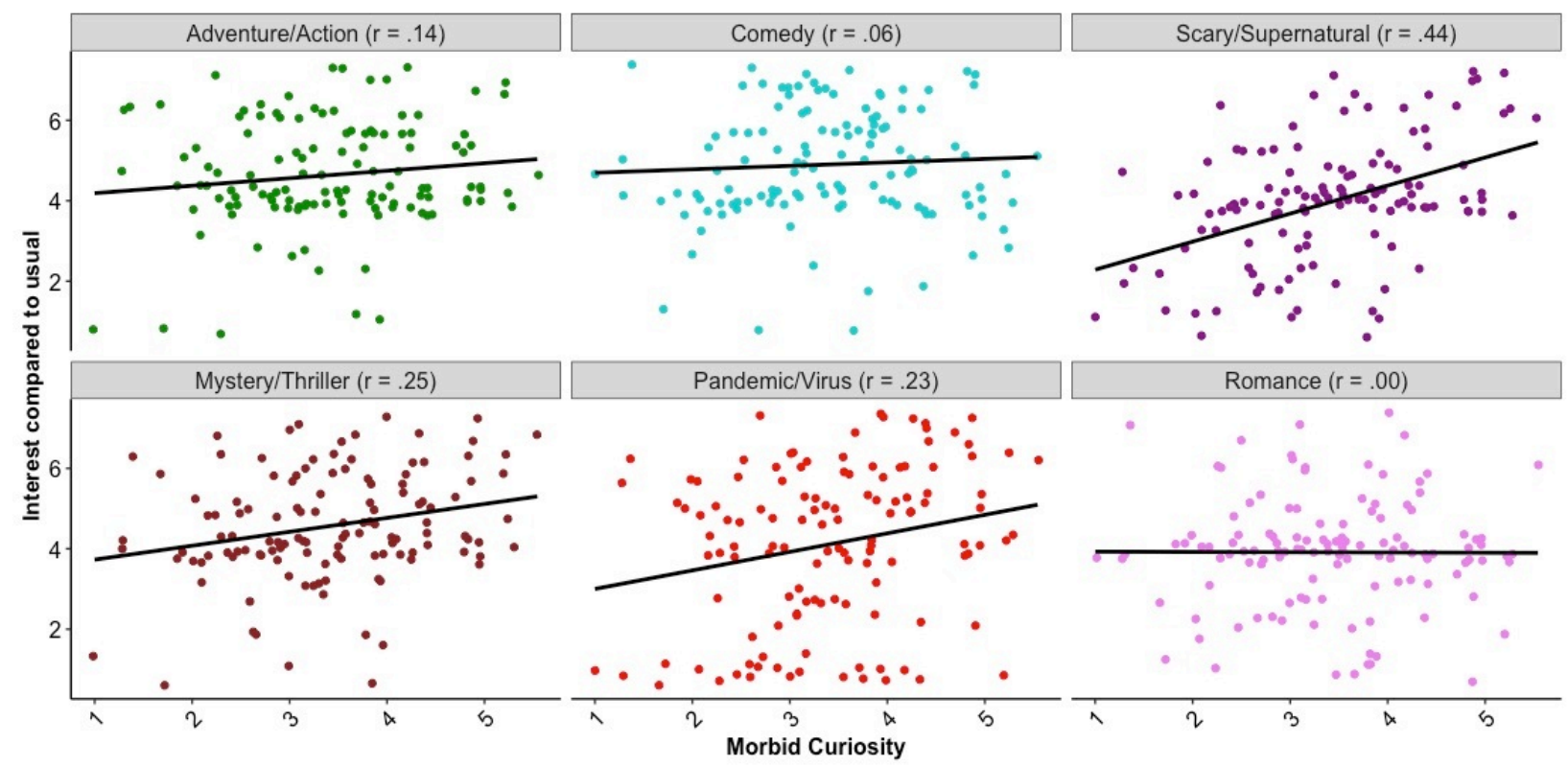

FIGURE 1 Correlation between trait morbid curiosity and interest in watching a movie or TV show from each genre in the next week compared to usual (1 - Much less interested, 7 - Much more interested).

\section{TABLE 1}

Descriptive Statistics for Coronavirus Interest and Media Preferences Questions

Questions
How interested are you in watching a pandemic or virus
movie or TV show in the next week compared to usual?

How interested are you in watching a scary / supernatural movie or TV show in the next week compared to usual?

$M \quad S D$

How interested are you in watching a mystery / thriller movie or TV show in the next week compared to usual?

How interested are you in watching an adventure / action movie or TV show in the next week compared to usual?

How interested are you in watching a watching comedy movie or TV show in the next week compared to usual?

How interested are you in watching a romance movie or TV show in the next week compared to usual?

How interested are you in learning what Coronavirus does to the human body?

How interested are you in learning about how Coronavirus is influencing policy? 
How interested are you in learning about Coronavirus death rates and countries that are impacted the most?

How interested are you in hearing stories about people who have died from Coronavirus?

How interested are you in hearing stories about people who have recovered from Coronavirus?

How interested are you in learning about the CDC guidelines for lowering your risk of Coronavirus infection?

*How long do you spend each day learning about Coronavirus, including reading/watching news about it?

How curious are you about the morbid aspects of Coronavirus?

How interested would you be in seeing photos of what Coronavirus does to the body?

How interesting do you think Coronavirus is?

How threatened do you feel by Coronavirus?

\section{$5.42 \quad 1.44$}

$3.92 \quad 1.77$

$4.77 \quad 1.77$

$5.30 \quad 1.59$

$2.53 \quad 1.2$

$3.94 \quad 2.04$

$3.67 \quad 2.09$

$5.06 \quad 1.63$

$4.62 \quad 1.68$

${ }^{*}$ This was a 5-point scale for clarity in answer-choice (1 - less than one hour per day, 5 - more than 3 hours per day; All other answer-choices were on 7-point scales (1 - Not at all, 7 - Extremely, for Coronavirus questions; 1 Much less interested, 7 - Much more interested, for genre questions).

\section{Exploratory Factor Analysis}

Data were screened for multivariate assumptions (normality, linearity, homogeneity, and homoscedasticity) to ensure they were appropriate for exploratory factor analysis. One outlier was detected using Mahalanobis distance $\left(X^{2}(10)=29.59\right)$ and was removed from further analysis. Bartlett's test indicated correlation adequacy $\left(X^{2}(45)=476.24, p<.001\right)$ and the Kaiser-Meyer-Olkin (KMO) test indicated sampling adequacy for exploratory factor analysis $(M S A=0.79)$.

A parallel analysis suggested a two-factor model of morbid curiosity. Maximum likelihood estimation was used with direct oblimin (oblique) rotation to examine factor structure. Using a factor loading criterion of .40, all items except for the news item loaded on only one factor. After the question asking how long the participant spent each day learning about Coronavirus from the news was removed from analysis, the model achieved simple structure with each of the items loading on only one of the factors. Item loadings ranged from .47 to .89. The first factor consisted of three items: interest in seeing photos of what Coronavirus does to the body, interest in what Coronavirus does to the human body, and interest in the morbid aspects of Coronavirus. Responses to these three measures were averaged and combined into a "morbid interest in Coronavirus" variable (Cronbach's $a=.84$ ). The second factor consisted of the remaining six Coronavirus questions (Table 2). Responses to these measures were averaged and combined into a "general interest in Coronavirus" variable $(a=.77)$. The mean and standard deviation for each factor was: morbid interest $M=4.18(S D=1.71)$, general interest $M=4.98$ $(S D=1.08)$. 
TABLE 2

Factor Loadings for Coronavirus Interest Questions

\begin{tabular}{lcc} 
Questions & $\begin{array}{c}\text { Morbid } \\
\text { Interest }\end{array}$ & $\begin{array}{c}\text { General } \\
\text { Interest }\end{array}$ \\
\hline $\begin{array}{l}\text { How interested are you in learning about how } \\
\text { Coronavirus is influencing policy? }\end{array}$ & -0.21 & $\mathbf{0 . 5 4}$ \\
$\begin{array}{l}\text { How interested are you in learning about Coronavirus } \\
\text { death rates and countries that are impacted the most? }\end{array}$ & 0.07 & $\mathbf{0 . 6 7}$ \\
$\begin{array}{l}\text { How interested are you in hearing stories about people } \\
\text { who have died from Coronavirus? }\end{array}$ & 0.29 & $\mathbf{0 . 4 9}$ \\
$\begin{array}{l}\text { How interested are you in hearing stories about people } \\
\text { who have recovered from Coronavirus? }\end{array}$ & -0.03 & $\mathbf{0 . 6 9}$ \\
$\begin{array}{l}\text { How interested are you in learning about the CDC } \\
\text { guidelines for lowering your risk of Coronavirus } \\
\text { infection? }\end{array}$ & $\mathbf{- 0 . 1 6}$ & $\mathbf{0 . 7 3}$ \\
$\begin{array}{l}\text { How interesting do you think Coronavirus is? } \\
\text { How curious are you about the morbid aspects of } \\
\text { Coronavirus? }\end{array}$ & 0.21 & $\mathbf{0 . 0 9}$ \\
$\begin{array}{l}\text { How interested would you be in seeing photos of what } \\
\text { Coronavirus does to the body? }\end{array}$ & $\mathbf{0 . 8 9}$ & \\
$\begin{array}{l}\text { How interested are you in learning about what } \\
\text { Coronavirus does to the human body? }\end{array}$ & $\mathbf{0 . 6 1}$ & -0.08
\end{tabular}

\section{Morbid curiosity, disgust sensitivity, threat, and interest in Coronavirus}

Neither trait morbid curiosity $(p=.092)$ nor disgust sensitivity $(p=.089)$ were significantly related to general interest in Coronavirus. Disgust sensitivity was also unrelated to morbid interest in Coronavirus $(p=.520)$. However, morbid curiosity was significantly positively correlated with morbid interest in Coronavirus $(p<.001$; Figure 2$)$.

New models were constructed with disgust sensitivity, morbid curiosity, and how threatened the participant felt by Coronavirus as predictors. Of these three predictors, only how threatened the participant felt by Coronavirus predicted general interest in Coronavirus $(p<$ $.001)$. However, both morbid curiosity $(\beta=0.98, S E=0.14, p<.001)$ and how threatened the participant felt by Coronavirus $(\beta=0.26, S E=0.08, p=.002)$ predicted morbid interest in Coronavirus. See Table 3 for all regression statistics. 


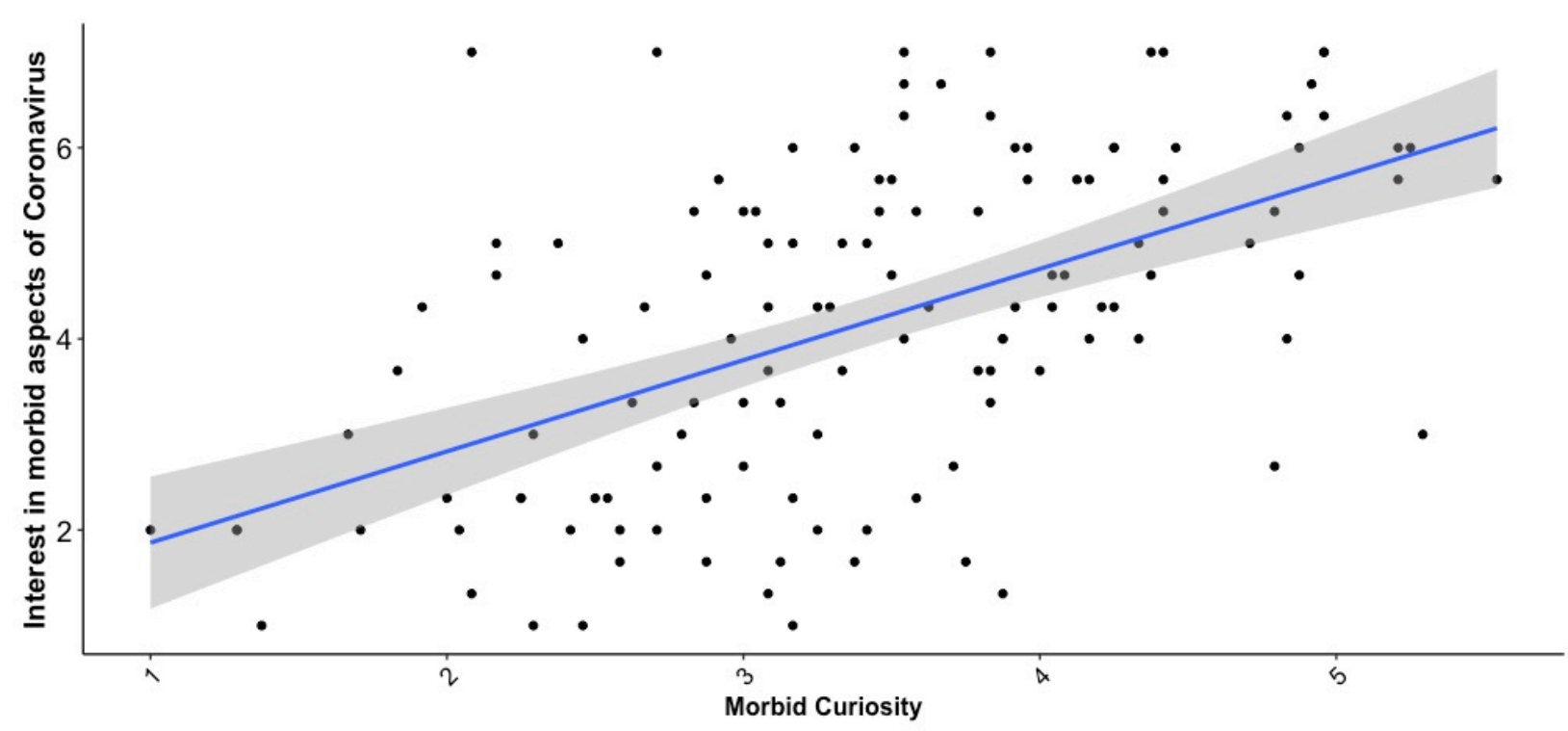

FIGURE 2 Correlation between morbid curiosity and morbid interest in Coronavirus ( $\mathrm{r}=$ $.54)$.

TABLE 3

Linear Regression Models Controlling for Sex and Age

\begin{tabular}{lcccc}
\hline Morbid Curiosity and Media Interests & $\boldsymbol{\beta}$ & $\boldsymbol{S E}$ & $\boldsymbol{t}$ & $\boldsymbol{p}$ \\
\hline Interest in pandemic/virus media compared to usual & 0.38 & 0.17 & 2.20 & $\mathbf{. 0 3 0}^{*}$ \\
Interest in scary/supernatural media compared to usual & 0.71 & 0.13 & 5.43 & $<. \mathbf{0 0 1}^{* * *}$ \\
Interest in mystery/thriller media compared to usual & 0.37 & 0.13 & 2.99 & $\mathbf{. 0 0 3}^{* * *}$ \\
Interest in adventure/action media compared to usual & 0.21 & 0.12 & 1.70 & .091 \\
Interest in comedy media compared to usual & 0.14 & 0.13 & 1.06 & .291 \\
Interest in romance media compared to usual & 0.04 & 0.13 & 0.28 & .778 \\
\hline Disgust sensitivity and Media Interests & & & & \\
\hline Interest in pandemic/virus media compared to usual & 0.07 & 0.28 & 0.25 & .804 \\
Interest in scary/supernatural media compared to usual & -0.30 & 0.23 & -1.31 & .193 \\
Interest in mystery/thriller media compared to usual & -0.11 & 0.21 & -0.55 & .587 \\
Interest in adventure/action media compared to usual & -0.51 & 0.20 & -2.58 & $\mathbf{. 0 1 1}$ \\
Interest in comedy media compared to usual & 0.12 & 0.21 & 0.59 & .559 \\
Interest in romance media compared to usual & 0.15 & 0.20 & 0.75 & .456 \\
\hline Interest in Coronavirus - Separate Models & & & & \\
\hline Morbid interest $\sim$ Morbid curiosity & 0.93 & 0.14 & 6.81 & $<.001 * * *$ \\
Morbid interest $\sim$ Disgust sensitivity & 0.17 & 0.26 & 0.65 & .520 \\
General interest $\sim$ Morbid curiosity & 0.15 & 0.10 & 1.49 & .138
\end{tabular}




\begin{tabular}{lcccc} 
General interest $\sim$ Disgust sensitivity & 0.30 & 0.16 & 1.88 & .063 \\
\hline Morbid Interest in Coronavirus - All Predictors in Model & & & \\
\hline Morbid curiosity & 0.98 & 0.14 & 7.17 & $<\mathbf{. 0 0 1}^{* * *}$ \\
Disgust sensitivity & 0.38 & 0.23 & 1.62 & .108 \\
Feeling threatened by Coronavirus & 0.26 & 0.08 & 3.13 & $\mathbf{. 0 0 2}^{* * *}$ \\
\hline General Interest in Coronavirus - All Predictors in Model & & & \\
\hline Morbid curiosity & 0.13 & 0.09 & 1.36 & .176 \\
Disgust sensitivity & 0.04 & 0.16 & 0.24 & .813 \\
Feeling threatened by Coronavirus & 0.34 & 0.06 & 6.16 & $<\mathbf{. 0 0 1}^{* * * *}$ \\
\hline
\end{tabular}

$* \mathrm{p}<.050 .{ }^{* *} \mathrm{p}<.010 .{ }^{* * *} \mathrm{p}<.001$.

\section{DISCUSSION}

Pandemics provide a unique opportunity to study how individuals process and respond to cues of a novel threat in their environment. In the midst of the 2020 Coronavirus pandemic, participants in this study reported their interest in a variety of topics related to Coronavirus as well as their interest now, compared to usual, in six genres of movies and television. These reports were analyzed in relation to three main predictor variables: trait morbid curiosity, trait disgust sensitivity, and how threatened the participant felt by Coronavirus. The primary purpose of the study was to investigate how trait morbid curiosity was related to current interest in both morbid and general information about Coronavirus, whether this relationship was present when considering both factual and fictional information, and whether or not morbid curiosity was related to interest in threatening information more broadly.

Morbidly curious individuals reported more interest than non-morbidly curious individuals in learning specifically about the morbid aspects of the virus, such as seeing photos of what Coronavirus does to the body. By promoting the seeking of factual information about the gritty details of a threat, morbid curiosity may lead some individuals to be more broadly informed about that threat. Interestingly, morbid curiosity was unrelated to how threatened one feels by Coronavirus. This may indicate that the psychological mechanisms behind morbid curiosity operate by increasing the seeking of morbid features rather than affecting how threatening they are perceived to be. Although increased feelings of threat toward Coronavirus led to more information gathering about it, this appeared to be independent from morbid curiosity. Even though morbid curiosity may be defined as an interest in threatening features of the world, the expression of morbidly curious behaviors might be unrelated to the degree of threat felt by the individual. Future studies should work to parse out the emotional and personality correlates of feeling morbidly curious about some phenomenon as a way to better understand the function and psychological mechanisms of morbid curiosity. In particular, why do morbidly curious individuals specifically seek out morbid information about some threat rather than information about the threat more broadly?

In addition to factual information, people can seek out simulated experiences of situations in the form of movies and TV shows. During the Coronavirus pandemic, morbidly curious participants in this study reported more interest than usual in pandemic movies and TV shows. One explanation for this finding is that the Coronavirus pandemic signified an imminent threat, 
leading individuals who were high in morbid curiosity to become particularly interested in gathering information about pandemics and viruses through fictional simulations. While this may explain why morbidly curious individuals were more interested in morbid information about Coronavirus and more interested in watching pandemic and virus films and TV shows, it does not explain why morbidly curious participants also reported greater interest in scary/supernatural and mystery/thriller genres. Increased interest in these genres may imply that morbidly curious individuals felt more interested in information about threats more broadly in response to the increased salience of the Coronavirus threat. However, another possibility is that the broader interest in scary/supernatural and mystery/thriller genres among morbidly curious individuals during the pandemic is due to escapism. Because morbidly curious individuals are generally more tolerable of and even drawn toward morbid phenomena, they may find morbid entertainment more amenable as a form of escapism while they are quarantined at home, whereas those who are less morbidly curious may partake in escapism via other genres.

Another intriguing possibility is that participants may not have considered some popular horror films and TV shows, such as 28 Days Later or The Walking Dead, to be in the pandemic/virus genre (but did consider them to be scary/supernatural) even though they provide cues of pathogen threat and information that could be relevant for a viral pandemic. The premise of both 28 Days Later and The Walking Dead, among other zombie films, is what happens to humans individually and society more broadly during a dangerous viral outbreak. Both fictions include information about infection, gathering supplies, and how people will (or will not) work together during a deadly pandemic. Although these fictions are probably not categorized as pandemic or virus films by many people, they would be excellent simulations for understanding the dynamics of viral pandemics. Thus, the increased interest in horror and thriller genres among the morbidly curious may have been the result of some popular examples of horror and thriller genres tapping into the same psychological mechanisms as viral pandemic films like Contagion and Outbreak. Future studies might investigate whether or not horror and thriller films that specifically provide simulations of pandemics inspire greater interest among morbidly curious individuals during real-life pandemics. One specific genre that might see an increase in interest is the zombie genre, since zombie outbreaks almost always occur in the form of a viral pandemic.

Finally, the importance of learning about the Coronavirus pandemic through news or fiction may have far outweighed the perceived costs for most people, not just for morbidly curious individuals. This could have led less morbidly curious individuals to also invest time in learning about the virus, resulting in a dampened association between general interest in Coronavirus and trait morbid curiosity. Since data collection was conducted three months after the beginning of the outbreak, it is also possible that morbidly curious participants had already satisfied their curiosity with pandemic-specific media, and thus their interest in watching more in the next week was not as high as it would have been earlier in the pandemic. Future studies should take advantage of longitudinal sampling to see how morbid curiosity relates to changes in media preferences in response to pandemics as well as other global and local events such as wars or increases in local crime. It will be interesting to see if morbid curiosity leads some individuals to seek out entertainment concerned specifically with the relevant threat, or, alternatively, to seek out threatening entertainment more broadly.

Another trait that might be predicted to have an influence on media preferences about a virus during a pandemic would be disgust sensitivity. However, in this study, disgust sensitivity was neither associated with general interest nor morbid interest in Coronavirus. This indicates that trait morbid curiosity may be a better predictor for disgusting and morbid news preferences 
than disgust sensitivity. Likewise, disgust sensitivity was unrelated to increased interest in pandemic films and TV shows, suggesting that changes in interest in that specific genre among some participants is better explained by morbid curiosity. Feeling threatened by Coronavirus was positively correlated with disgust sensitivity and more interest in both morbid and general information about Coronavirus. However, greater disgust sensitivity was not significantly associated with interest in either general or morbid information about Coronavirus. This may mean that the psychological mechanisms underlying disgust sensitivity primarily act on behaviors other than information gathering, while those underlying perceived threat from disgust act more directly on information gathering. However, these speculations should be taken with caution. The threat measure was a single question that may not have captured the full breadth of how one does or does not feel threatened by Coronavirus. Future research should aim to further disentangle the relationship between disgust sensitivity, threat-perception, and morbid curiosity.

The present study only explored a small part of how trait morbid curiosity relates to media preferences, and several traits that might be related to morbid curiosity and relevant to media preferences were not included in the study. Two traits that should be analyzed in relation to morbid curiosity in future research are sensation seeking and novelty seeking. Sensation seeking is a well-established trait defined by the "seeking of varied, novel, complex, and intense sensations and experiences, and willingness to take physical, social, legal, and financial risks for the sake of such experience" (Zuckerman 1994, p. 27). One early study on morbid curiosity found that it was positively related to sensation seeking (Zuckerman and Litle 1986). However, a more recent study that relied on behavioral demonstrations of morbid curiosity found that the reported intensity of a negative image was unrelated to participants' curiosity about the image, casting some doubt on the relationship between sensation seeking and morbid curiosity (Oosterwijk 2017). In a meta-review, Hoffner and Levine (2005) found that sensation seeking was significantly correlated with enjoyment of frightening and violent media, but the effect size was relatively small $(\mathrm{r}=.20)$. In another study, thrill seeking, a trait related to sensation seeking, explained about $25 \%$ of the variance in scores on the Morbid Curiosity Scale (Scrivner 2020). In the same study, openness to experience, a trait related to novelty seeking, only explained about $1 \%$ of the variance in trait morbid curiosity. While it seems clear that many morbid features of the world are often both sensational and novel, the relationship between trait morbid curiosity and trait sensation and novelty seeking remains unclear. Future research should investigate how these traits and others (e.g., anxiety, neuroticism) relate to morbid curiosity.

Another interesting avenue of research would be to investigate morbid curiosity and interest in other types of morbid entertainment, such as horror-themed video games or haunted attractions. Trait morbid curiosity may also play a role in tourism. In particular, individuals high in morbid curiosity may tend toward "thanatourism" or "dark tourism" - attractions that relate to death and disaster (Podoshen, Venkatesh, Wallin, Andrzejewski, and Jin 2015). Finally, it is important to investigate how trait morbid curiosity relates to baseline interests as well as differences in reactivity - that is, do morbidly curious people simply have a higher baseline interest in morbid material, or, as this study suggests, is their morbid curiosity also more reactive to morbid events? Further exploration of how trait morbid curiosity relates to entertainment preferences could shed light on the psychology of this understudied trait and how it relates to broader human behaviors that involve death, including rituals, religions, and myths.

\section{WORKS CITED}

Ariely, Dan, and George Loewenstein. 2006. "The heat of the moment: The effect of sexual 
arousal on sexual decision making." Journal of Behavioral Decision Making 19, no. 2: 87-98. doi:10.1002/bdm.501.

Clasen, Mathias, Jens Kjeldgaard-Christiansen, and John A. Johnson. 2018. "Horror, personality, and threat simulation: A survey on the psychology of scary media." Evolutionary Behavioral Sciences (2018). doi:10.1037/ebs0000152.

Google Trends. 2020. Google Trends. [online] Available at: <https://www.google.com/trends/> [Accessed March 20, 2020].

Haidt, Jonathan, Clark McCauley, and Paul Rozin. 1994. "Individual differences in sensitivity to disgust: A scale sampling seven domains of disgust elicitors." Personality and Individual differences 16, no. 5:701-13. doi:10.1016/0191-8869(94)90212-7.

Hoffner, Cynthia A., and Kenneth J. Levine. 2005. "Enjoyment of mediated fright and violence: A meta-analysis." Media Psychology 7, no. 2:207-37. doi:10.1207/S1532785XMEP0702 5.

Ibarra, Frank, and Dario Maestripieri. 2017. "Assessing people's interest in images with violent or disgusting content: a functional-evolutionary analysis." Evolutionary Psychological Science 3, no. 2:133-40. doi:10.1007/s40806-016-0082-4.

Mack, David. "Everyone is Watching "Contagion," A 9-Year-Old Movie about a Flu Outbreak." BuzzFeed News, March 3, 2020. Accessed March 6, 2020. https://www.buzzfeednews.com/article/davidmack/contagion-movie-coronavirus.

Mar, Raymond A., and Keith Oatley. 2008. "The function of fiction is the abstraction and simulation of social experience." Perspectives on psychological science 3, no. 3:173-92. doi:10.1111/j.1745-6924.2008.00073.x

Morin, Olivier, Alberto Acerbi, and Oleg Sobchuk. 2019. "Why people die in novels: testing the ordeal simulation hypothesis." Palgrave Communications 5, no. 1:1-10. doi:10.1057/s41599-019-0267-0.

Öhman, Arne, and Susan Mineka. 2001. "Fears, phobias, and preparedness: toward an evolved module of fear and fear learning." Psychological review 108, no. 3: 483-522. doi:10.1037/0033-295x.108.3.483.

Olatunji, Bunmi O., Nathan L. Williams, David F. Tolin, Jonathan S. Abramowitz, Craig N. Sawchuk, Jeffrey M. Lohr, and Lisa S. Elwood. 2007. "The Disgust Scale: item analysis, factor structure, and suggestions for refinement." Psychological assessment 19, no. 3:281-97. doi:10.1037/1040-3590.19.3.281.

Oosterwijk, Suzanne. 2017. "Choosing the negative: A behavioral demonstration of morbid curiosity." PloS one 12, no. 7. doi:10.1371/journal.pone.0178399.

Peer, Eyal, Laura Brandimarte, Sonam Samat, and Alessandro Acquisti. 2017. "Beyond the Turk: Alternative platforms for crowdsourcing behavioral research." Journal of Experimental Social Psychology 70:153-63. doi:10.1016/j.jesp.2017.01.006.

Piaget, Jean. 1945. Play, Dreams, and Imitation in Childhood. New York: Norton Library.

Podoshen, Jeffrey S., Vivek Venkatesh, Jason Wallin, Susan A. Andrzejewski, and Zheng Jin. 2015. "Dystopian dark tourism: An exploratory examination." Tourism Management 51: 316-328. doi:10.1016/j.tourman.2015.05.002.

Schaller, Mark. 2011. "The behavioural immune system and the psychology of human sociality." Philosophical Transactions of the Royal Society B: Biological Sciences 366, no. 1583:3418-26. doi:10.1098/rstb.2011.0029.

Scrivner, Coltan. 2020. "The Psychology of Morbid Curiosity." PsyArXiv. doi:10.31234/osf.io/xug34. 
Scrivner, Coltan, Kyoung Whan Choe, Joseph Henry, Muxuan Lyu, Dario Maestripieri, and Marc G. Berman. 2019. "Violence reduces attention to faces and draws attention to points of contact." Scientific reports no. 9:17779. doi:10.1038/s41598-019-54327-3.

Stevenson, Richard J., Trevor I. Case, and Megan J. Oaten. 2011. "Effect of self-reported sexual arousal on responses to sex-related and non-sex-related disgust cues." Archives of sexual behavior 40, no. 1:79-85. doi: 10.1007/s10508-009-9529-z.

Tybur, Joshua M., Debra Lieberman, Robert Kurzban, and Peter DeScioli. 2013. "Disgust: Evolved function and structure." Psychological review 120, no. 1:65-84. doi:10.1037/a0030778.

Zuckerman, Marvin. 1994. Behavioral expressions and biosocial bases of sensation seeking. Cambridge university press

Zuckerman, Marvin, and Patrick Litle. 1986. "Personality and curiosity about morbid and sexual events." Personality and Individual Differences 7, no. 1:49-56. doi:10.1016/01918869(86)90107-8. 